\title{
PESQUISA MICOTOXICOLÓGICA EM FÍGADO DE AVES PRODUZIDAS E COMERCIALIZADAS EM PERNAMBUCO
}

\author{
ELISA ALVES VILAR * \\ MARIA CRISTINA MONTEIRO OLIVEIRA ** \\ TÂNIA LÚCIA MONTENEGRO STAMFORD ***
}

\begin{abstract}
Com o objetivo de verificar a incidência de micotoxinas em fígados de aves abatidas em regiões do Estado de Pernambuco foram analisados 40 fígados procedentes da zona litorânea e 33 fígados da zona da mata. Cinqüenta por cento (50\%) das amostras foram classificadas como próprias para o consumo e o restante como impróprias por apresentarem coloração amarelada, estrutura friável e tamanho aumentado. As amostras foram divididas em 4 grupos e classificadas como: amostra 1 - fígado íntegro Zona da Mata (11 amostras); amostra 2 - fígado condenado Zona da Mata (22 amostras); amostra 3 - fígado íntegro Zona Litorânea (20 amostras); amostra 4 - fígado condenado Zona Litorânea (20 amostras). Os fígados, após resfriamento foram analisados pelo ensaio imunoenzimático (ELISA) e por Cromatografia em Camada Delgada (CCD). Pelo método ELISA foi pesquisada aflatoxina total e pelo método CCD aflatoxina B1, B2, G1 e G2, zearalenona e ocratoxina A. Os resultados obtidos mostraram incidência de contaminação por aflatoxinas (B1, B2, G1 e G2) em todas as amostras em concentrações inferiores a 5 ppb (limite de quantificação do método), nível compatível com a legislação brasileira. As amostras $1,2,3$ e 4 analisadas pelo ensaio imunoenzimático (ELISA) evidenciaram os seguintes resultados: 3,8, 4,2, 4,7 e 5,2 ppb de aflatoxinas, respectivamente. Zearalenona e ocratoxina $A$ não foram detectadas. Esses resultados demonstram que a pesquisa micotoxicológica em produtos avícolas serve como subsídio para monitoramento a ser realizado pelo setor produtivo, capaz de minimizar perdas de produção e proporcionar produto avícola menos contaminado por micotoxinas.
\end{abstract}

PALAVRAS-CHAVE: FÍGADO DE AVES; AFLATOXINAS; OCRATOXINA A, ZEARALENONA.

* Médica Veterinária, Doutoranda, Departamento de Nutrição, Universidade Federal de Pernambuco (UFPE), Parnamirim, Recife - PE. (e-mail: aureo@elógica.com.br).

** Química, Divisão de Toxicologia do Laboratório Central de Saúde Pública do Estado de Pernambuco (DITOX-LACEN-PE), Boa Vista, Recife - PE.

*** Prof ${ }^{a}$. Adjunta, Departamento de Nutrição, UFPE, Cidade Universitária, Recife - PE. 


\section{INTRODUÇÃO}

A carne de aves constitui uma das mais consumida por todas as camadas da população e Pernambuco representa 4,85\% da produção nacional. É o primeiro produtor do Nordeste (APINCO, 1996) e a avicultura é a primeira economia do Estado (AVIPE, 2000). A ração das aves é composta principalmente de milho (60\%) e de soja (20\%) e nesses ingredientes, micotoxinas têm sido isoladas constantemente. Pouco se conhece sobre resíduos de micotoxinas em produtos avícolas produzidos e comercializados nacionalmente, com exceção de aflatoxina que já foi isolada em ovos (FRAGA et. al., 1996) e em fígado de aves (LORENZINI, 1997) naturalmente contaminadas, além de isolamentos experimentais. Segundo SABINO (2000) a ingestão da ração contaminada proporciona a biotransformação e transmissão das aflatoxinas. Ressaltou também que essa segunda via de contaminação é preocupante, principalmente, quando se trata de produtos de origem animal, entre os quais carnes e ovos.

Entre as vísceras comestíveis de aves, o fígado é economicamente mais importante, sendo bastante utilizado como matéria-prima para processados (patês). Segundo LORENZINI (1997), no Rio Grande do Sul, durante as quatro estações do ano de 1996 ocorreu o isolamento de aflatoxina (no fígado) em plantéis avícolas.

O Brasil é o segundo produtor mundial de milho, cuja safra 2000/2001 totalizou 38.497,3 toneladas (CONAB, 2001), porém essa produção não é suficiente para o abastecimento interno (CORREA et al., 1998). Por tratar-se de cultura com grande susceptibilidade à contaminação fúngica (desde a floração), constituir alimento bem aceito pela população e devido seu uso em rações (CRUZ, 1995), o milho tem sido monitorado.

A maior produção de milho do Brasil encontra-se nos Estados de São Paulo e Minas Gerais, cujas condições climáticas favorecem o crescimento fúngico. Nas Regiões Sul e Sudeste verifica-se alternância de noites frias com dias quentes e oscilações de temperatura diárias e noturnas. Práticas agrícolas como deixar o milho colhido no campo para secar favorece o desenvolvimento de fungos como o Fusarium. Esses produzem toxinas sob efeito de choque térmico, como a queda de temperatura que ocorre à noite (SCUSSEL, 1998a).

As micotoxinas causam imunodepressão e interferem diretamente nos parâmetros produtivos das aves, podendo levá-las a morte (SFOGGIA e SALLE, 1998). A contaminação freqüente dos grãos causa exposição 
crônica à toxina via ração contaminada. As perdas causadas por micotoxinas, segundo estimativa da FAO, são mundiais e situam-se ao redor de $25 \%$ dos grãos produzidos (VIEIRA, 1995). Na avicultura industrial, rações contaminadas mesmo com doses inferiores a $75 \mathrm{ppb}$ de aflatoxina provocam reduções de até 10\% no peso das aves (LAZZARI, 1997).

A presença de aflatoxinas em alimentos foi regulamentada no Brasil, em 1976, pela Resolução no 34/76 da Comissão Nacional de Normas e Padrões para Alimentos (BRASIL, 1977), que prevê o máximo de 30 ppb (SILVA et. al., 1996). A legislação sobre aflatoxina refere-se aos produtos naturais e não contempla as demais micotoxinas. Porém, vários governos já instituíram limites regulamentares para micotoxinas em alimentos e rações animais para venda ou importação. Os países do MERCOSUL estabeleceram a Resolução $n-56 / 94$, que foi internalizada pela Portaria no183 (de 21/3/96) do Ministério de Agricultura e Abastecimento. A Resolução no 56/94 do MERCOSUL determina o máximo de 20 ppb para o somatório das aflatoxinas $\mathrm{B} 1+\mathrm{B} 2+\mathrm{G} 1+\mathrm{G} 2$, desde que a aflatoxina B1 seja inferior a $15 \mathrm{ppb}$. Para o leite permite limite máximo de $0,5 \mathrm{ppb}$ de aflatoxina M1. Em alimentos infantis aflatoxinas não devem ser detectadas, e nas rações animais é permitido no máximo 100 ppb (SILVA et. al., 1996). Esses limites estabelecidos no âmbito do MERCOSUL são compatíveis com outros países. Quanto às outras micotoxinas, existem discussões sobre a zearalenona, a ocratoxina $A$ e a patulina. Os tricotecenos constam da lista de prioridades e para fumonisina ainda não há dados suficientes.

Este trabalho teve por objetivo a pesquisa micotoxicológica em fígado de aves produzidas e comercializadas em regiões do Estado de Pernambuco, utilizando-se dois métodos de análise.

\section{MATERIAL E MÉTODOS}

Foram coletadas 73 amostras fígado de aves, em dois abatedouros industriais, situados na Zona da Mata (33 fígados) e no Litoral (40 fígados), as duas maiores regiões produtivas do Estado de Pernambuco. Os dois abatedouros foram escolhidos por serem os maiores das duas regiões.

As amostras foram classificadas como íntegras (própria para o consumo) e condenadas por apresentarem características macroscópicas (cor, volume e consistência) indesejáveis para a comercialização e divididas em 4 grupos: amostra 1 - fígado íntegro Zona da Mata (11 amostras); 
amostra 2 - fígado condenado Zona da Mata (22 amostras); amostra 3 fígado íntegro Zona Litorânea (20 amostras); amostra 4 - fígado condenado Zona Litorânea (20 amostras).

As aves foram produzidas em diferentes granjas situadas nas duas regiões estudadas, cujo período de crescimento (início de abril ao final de março de 2000) correspondeu a época das chuvas e portanto com maior desenvolvimento fúngico.

Em cada região de produção (zona da mata e litoral) a empresa avícola de grande porte, proprietária do abatedouro, fornece os pintos de um dia, assistência técnica e ração balanceada aos produtores rurais pelo sistema de produção Integrado (fornecedor/produtor). Dessa forma, as aves foram alimentadas com rações industriais de duas fontes de grãos, uma de cada região.

Ao final do crescimento as aves foram abatidas, evisceradas e os fígados após resfriamento, analisados no Laboratório de Patologias Avícolas de Pernambuco (LAPAS-PE) e na Divisão de Toxicologia do Laboratório Central de Saúde Pública do Estado de Pernambuco (DITOX-LACEN-PE).

Utilizou-se apenas um lóbulo do fígado (desprezando-se a vesícula biliar) fragmentado em três partes iguais (colocadas em recipientes separados), visando obtenção de três amostras coletivas para analisar por dois métodos e uma como contraprova. Nas amostras de fígado enviadas para o LAPAS$\mathrm{PE}$ analisou-se aflatoxina total. Na extração da micotoxina foram utilizados $50 \mathrm{~g}$ da amostra coletiva e $250 \mathrm{~mL}$ de metanol a $70 \%$ como extrator. Homogeneizou-se o produto por 30 minutos em stomacher, filtrando-se em seguida em papel de filtro quantitativo. Essa metodologia foi desenvolvida para análise de ração e descrita por SALLE et. al. (1994) para extração de aflatoxinas em fígado de aves. Na detecção, utilizou-se o ensaio imunoenzimático ELISA (Enzyne Linked Immunosorbent Assay), segundo a recomendação do kit comercial Veratox da empresa Norteamericana Neogen Co. que emprega anticorpos monoclonais. Foram retirados $100 \mathrm{~mL}$ da amostra e procedeu-se a análise quantitativa. Nas amostras de fígado analisadas na DITOX-LACEN-PE foi utilizado o método desenvolvido por SOARES e RODRIGUES-AMAYA (1989) para a extração e quantificação das micotoxinas, com limite de detecção de 5.0 ppb para aflatoxinas (AFB1, AFB2, AFG1 e AFG2); 5,0 ppb para ocratoxina A e 60,0 ppb para zearalenona. Foi utilizada placa pronta de Sílica Gel G 60, com $0,25 \mathrm{~mm}$ de espessura (Wathman) como fase estacionária. Para eluição das toxinas foi empregado o sistema solvente tolueno : acetato 
de etila : ácido fórmico na proporção de 1:1:1. A visualização da presença da toxina padrão e nos extratos de amostras ocorreu sob luz ultravioleta de ondas longas e curtas, comparando-se visualmente a intensidade de fluorescência entre os padrões de aflatoxinas e o extrato da amostra, sempre na mesma cromatoplaca.

\section{RESULTADOS E DISCUSSÃO}

As características macroscópicas como cor, consistência e volume são utilizados como critério de seleção para a comercialização "in natura" de fígado de aves. Nas amostras analisadas, $50 \%$ foram classificadas como íntegras (própria para o consumo) e o restante como impróprias (condenadas) por apresentarem cor pálida, consistência friável e aumento de volume (Figura 1). Essas características podem ser consideradas como efeitos primários das aflatoxicoses. A primeira alteração ocorre no tamanho dos órgãos internos, principalmente do fígado, depois na coloração e textura que os torna amarelados e friáveis (SANTÚRIO, 1997). CRUZ (1999) observou que na aflatoxicose aviária ocorrem alterações histopatológicas, com hepatócitos aumentados, além de generalizada proliferação de células nos ductos biliares. A acentuada infiltração de gordura depende da dose e do tempo de intoxicação, chegando o fígado a $68 \%$ de aumento. A síntese hepática de gordura e o transporte desta para outras áreas do organismo são seriamente afetados pela ação da AFB1 (MERKLEY et al., 1987).

\section{FIGURA 1 - AMOSTRAS DE FÍGADOS DE AVES DO MESMO PLANTEL EABATE}

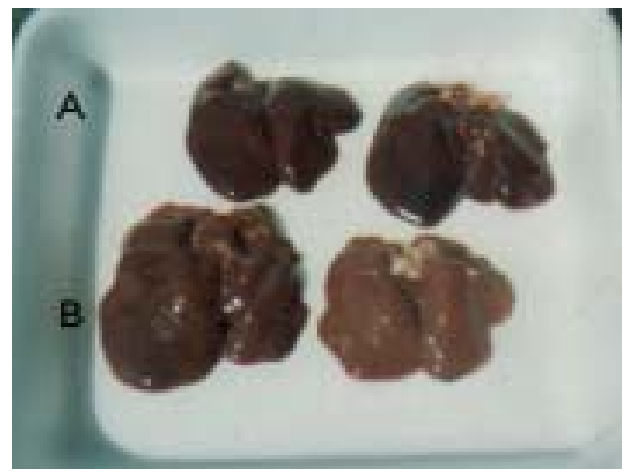

$\mathrm{A}=$ íntegros; $\mathrm{B}=$ impróprios para o consumo. 
Os resultados obtidos pela análise cromatográfica (CCD) mostraram contaminação por aflatoxinas (AFB1, AFB2, AFG1 e AFG2) em todas as amostras, considerados como traços pela concentração inferior a $5 \mathrm{ppb}$. A ocratoxina $A$ e a zearalenona não foram detectadas, o que permite inferir que as amostras apresentaram segurança para o consumidor. A presença de mais de uma micotoxina no alimento pode causar efeito acumulativo, tornando-o mais perigoso (VILAR e STAMFORD, 1998). Efeito sinérgico entre ocratoxinas e aflatoxinas foi verificado por SFOGGIA e SALLE (1998) em rações e rins de frango de corte, prejudicando a produção de empresa avícola do Rio Grande do Sul. O uso de mistura de grãos em rações possibilita a presença de mais de uma micotoxina.

As amostras 1, 2, 3 e 4 apresentaram pelo ensaio imunoenzimático (ELISA) resultados (3,8, 4,2, 4,7 e 5,2 ppb de aflatoxinas, respectivamente) abaixo do limite estabelecido pela legislação brasileira (BRASIL, 1977).

$\mathrm{Na}$ avicultura industrial, a contaminação por níveis reduzidos é de diagnóstico difícil, sem no entanto deixar de causar prejuízos econômicos. Como regra geral, aves jovens são mais sensíveis e aves em crescimento não devem ingerir mais de 20 ppb de aflatoxina na dieta.

Para o analista diversos fatores determinam e limitam a seleção do método apropriado (como por exemplo rapidez, simplicidade e baixo custo) para serem usados em análises rotineiras de inspeção. A utilização dos dois métodos de análise permitiu compara-los em relação à quantificação de aflatoxinas. Não houve diferença quantitativa entre os métodos, destacando-se maior precisão do ELISA. A técnica de CCD está intimamente ligada às análises de micotoxinas e apresenta significado histórico. Graças a CCD várias micotoxinas foram detectadas, isoladas e caracterizadas, desde as aflatoxinas até as fumonisinas (VASANTHIL e BHAT, 1998). Na década de 80 foi desenvolvido para a detecção de aflatoxinas, o ensaio imunoenzimático ELISA, logo consagrado como rápido, altamente sensível e de custo reduzido. No que se refere as micotoxinas é menos dispendioso do que muitos testes tradicionais, além de altamente seletivo para determinados tipos de toxinas. Esse sistema de detecção de micotoxinas é simples, dispensa equipamentos sofisticados, podem ser realizados praticamente a qualquer hora e lugar, contanto que a temperatura ambiente esteja entre 18 e $25^{\circ} \mathrm{C}$ segundo recomendação da Neogen Co. Assim, é ideal para ser usado em granjas mediante treinamento do usuário (SALLE et al., 2000).

Surtos de ocratoxicose em aves foram documentados, com nível de ocratoxina A variando de 3 a 16 ppm (ELISSALDE et al., 1994). Essa 
toxina reduz o ganho em peso, contribuí para problemas locomotores em frangos, induz condição similar à síndrome da ave pálida pela má absorção de nutrientes, causa imunodepressão e é principalmente nefrotóxica (GIBSON et al., 1990). Causa também lesões no fígado e aumento no seu volume (BORETTI, 1998). Apesar dessa característica macroscópica ter sido verificada em $50 \%$ das amostras, a ocratoxina A não foi detectada. CHOUDHURY et al. (1971) e DIRHEIMER (1996) verificaram que ausência da ocratoxina $A$ em sua forma metabolicamente ativa deve-se à sua rápida eliminação (poucos dias), após a remoção do alimento contaminado. SCUSSEL (1998a) verificou que ocratoxina A presente naturalmente em rações avícolas não deixa resíduo em tecidos, apesar de ter sido isolada em músculos de frango de corte em estudos experimentais.

Em relação à metodologia, SALLE et al. (2000) afirmaram que se pode monitorar a presença da ocratoxina A pelo ensaio imunoenzimático (ELISA) em vísceras de aves. Detectaram resíduos dessa toxina nos rins, em diferentes tempos pós-inoculação de uma só dose de 200 ppb de ocratoxina A diretamente no inglúvio. Tais autores também utilizaram o fígado juntamente com a vesícula biliar após a retirada da bile, e detectaram ocratoxina A. Porém no exame de amostras de bile dos animais foi observada pouca especificidade da prova de ELISA, uma vez que a ocratoxina $A$ foi detectada em altos níveis tanto nos animais quanto nos controles.

A exposição freqüente à zearalenona pode deixar resíduo dessa micotoxina em partes comestíveis de aves (em fígado por exemplo). É possível que não tenha havido contaminação freqüente nas rações dessas aves, ou talvez o nível de zearalenona consumido tenha sido baixo e por isso não detectado pelos métodos estudados. MARYAMMA et al. (1992) apud VIEIRA (1995) encontraram resíduos em fígado (207 ppb), rins (416 ppb), músculo (170 ppb) e moela (437 ppb) de frangas que consumiram $10 \mathrm{mg} /$ $\mathrm{kg}$ dessa toxina durante 20 dias. $\mathrm{O}$ ganho de peso foi superior ao dos animais controle o que demonstra o efeito estrogênico da substância. Os mesmos autores observaram resíduos apenas na forma de zearalenona. Altas concentrações de zearalenona parecem não ocasionar problemas sérios à produção de ovos e de carne de aves. Entretanto, deve-se chamar a atenção para a presença de metabólitos altamente estrogênicos nesses alimentos. O controle de zearalenona nos grãos deve ser considerado tão importante quanto o de outras micotoxinas causadoras de efeito visíveis (VIEIRA, 1995).

Na composição média das rações (ingredientes e níveis nutricionais) fornecidas às aves (Tabelas 1 e 2) verifica-se nível de $20 \%$ de proteína 
bruta, comumente usado pelos avicultores e suplementação de aminoácidos.

\section{TABELA 1 - COMPOSIÇÃO MÉDIA DAS RAÇÕES UTILIZADAS NO CRESCIMENTO DAS AVES}

\begin{tabular}{l|c}
\hline INGREDIENTE & $\%$ \\
\hline Milho & 61,50 \\
Farelo de Soja & 25,75 \\
leo de Soja & 3,00 \\
Farinha de carne & 5,00 \\
Fosfato Bic/Ecico & 2,00 \\
Calc/Fio & 0,90 \\
Sal iodado & 0,50 \\
Suplementa a vitam nica, & 0,10 \\
Suplementa a mineral † & 0,05 \\
DLmetionina (99\%) & 0,10 \\
Antioxidante & 0,50 \\
CoxidiostAfico ¥ & 0,10 \\
Inerte & 0,50 \\
TOTAL & 100,00 \\
\hline
\end{tabular}

1 Rovimix aves inicial (Roche) - Níveis de garantia por quilo do produto: Vitamina A - 12000000 UI; Vitamina D3 - 2500000 Ul; Vitamina E - 30000 Ul; Vitamina B1 - 2,0 g; Vitamina B6 - 3,0 g; Pantotenato de Cálcio - 10,0 g; Biotina - 0,07 g; Vitamina K3 - 3,0 g; Ácido Fólico - 1,0 g; Ácido Nicotínico - 35,0 g; Bacitracina de Zinco - 10,0 g; Cloreto de Colina - 100,0 g; BHT $-5,0 \mathrm{~g}$; Vitamina B12-15000 mg; Olaquindox - 5,0 g; Selênio -0,120 g.

2 Rologomix aves (Roche) - Níveis de garantia por quilo do produto: manganês - 160,0 g; Ferro - 100,0 g; Zinco - 100,0 g; Cobre - 20,0 g; Cobalto - 2,0 g; lodo - 2,0 g.

3 Pfizer Saúde Animal, São Paulo, SP.

4 Palha de arroz.

\section{TABELA 2 - NÍVEIS NUTRICIONAIS ENCONTRADOS NA RAÇÃO CRESCIMENTO}

\begin{tabular}{|c|c|}
\hline COMPOSI^O & N"VEIS DE GARANTIA \\
\hline Energia Metaboliz Ævel (kcal/kg) & $3.100,000$ \\
\hline Prote na $(\%)$ & 20,000 \\
\hline Metionina (\%) & 0,482 \\
\hline Metionina + Cistina $(\%)$ & 0,788 \\
\hline Lisina $(\%)$ & 1,150 \\
\hline С 圴cio (\%) & 0,992 \\
\hline $\mathrm{F}$ sforo dispon vel (\%) & 0,480 \\
\hline
\end{tabular}

No controle das intoxicações por micotoxinas, a suplementações de nutrientes, principalmente proteína, é recomendada. Dieta rica em aminoácidos sulfurados pode inibir os efeitos das aflatoxinas. Esses, ligam-se ao epóxido ativo da molécula de aflatoxina presente no fígado, tornando-a atóxica e passível de ser eliminada pela bile. Assim, as aves apresentam crescimento próximo ao de aves não intoxicadas (SCOTT, 
1993; CRUZ, 1995). Tal prática, de grande valia, torna-se inviável devido o custo/benefício quando são acrescentados índices maiores de aminoácidos que o normalmente usado nas rações.

\section{CONCLUSÃO}

A detecção de aflatoxina no fígado das aves, mesmo nos níveis encontrados, dimensiona o problema enfrentado pelos produtores avícolas e exige o estabelecimento de medidas de controle mais severas para rações e matérias-primas.

Os parâmetros produtivos, carne e ovos, são afetados pela ingestão constante da aflatoxina em níveis considerados baixos. Os resíduos provenientes dessa baixa contaminação, em partes comestíveis de aves, pressupõem que o consumidor esteja protegido. Mesmo assim, torna-se evidente a necessidade da implantação de programa de pesquisa de resíduo de micotoxinas, em partes comestíveis de aves, que além de minimizar perdas de produção possa servir como alerta da comunidade científica para os órgãos governamentais.

\section{Abstract}

\section{MICOTOXICOLOGICAL RESEARCH IN BIRD'S LIVER PRODUCED AND COMMERCIALIZED IN PERNAMBUCO}

With the objective of verifying the incidence of micotoxins in bird's livers abated in regions of the state of Pernambuco were analyzed 40 livers from Coast zone and 33 livers from Forest zone. Fifty percent (50\%) of these samples were classified good for consuming and the rest not good for consuming and the not good for consuming because of the yellowish co lour presented on them, friable structure and bigger size. The samples were divided in 4 groups and classified as: sample1 - healthy liver forest zone (11 samples); sample 2 - unhealthy liver forest zone (22 samples); sample 3 - healthy liver coast zone (20 samples); sample 4 - unhealthy liver coast zone (20 samples). The livers after being frozen, were analyzed by the methods ELISA and thin layer chromatography in (CCD). In the method (ELISA) it was researched total aflatoxin and in the method CCD it was researched aflatoxin B1, B2, G1 and G2; zearalenon and ocratoxin $A$. The results obtained revealed and incidence in the contamination by aflatoxins (B1, B2, G1 and G2) in all samples in concentrations lower than $5 \mathrm{ppb}$ (limit of quantification of method) being considered as the compatible level for the Brazilian legislation. The samples 1, 2, 3, and 4 analysed by the imunoenzymatic essay (ELISA) obtained the following results: $3.8,4.2,4.7$ and $5.2 \mathrm{ppb}$ of aflatoxins respectively. Zearalenon and ocratoxin A were analyzed by the CCD method and obtained negative results. These results demonstrated that the micotoxin research in bird's products serve as subsidy for the elaboration of a monitory to be realized by the productive sector, capable of minimize loss of production and supply to human consume of bird's product less contaminated by micotoxins.

KEY WORDS: BIRD'S LIVER; AFLATOXINS; OCHRATOXIN A; ZEARALENONE. 


\section{REFERÊNCIAS}

1 APINCO. Associação de Pintos de Corte do Estado de São Paulo. Quinze anos de levantamento APINCO. São Paulo: Associação Paulista de Avicultura, 1996. p. 9.

2 AVIPE. Associação Avícola de Pernambuco. Dados estatísticos 1999/2000. [Recife], 2001.

3 BORETTI, L. Micotoxinas em poedeiras. Revista Avicultura Industrial, São Paulo, n.1059, p. 41-44, set. 1998.

4 BRASIL. Ministério da Saúde. Comissão Nacional de Normas e Padrões para Alimentos. Resolução n. 34/76. Fixa padrões de tolerância para as aflatoxinas em alimentos. Diário Oficial [da] República Federativa do Brasil, Brasília, 19 janeiro 1977. Seção 1, pt. I, p. 710.

5 CHOUDHURY, H.; CARLSON, D. W.; SEMWNIUK, G. A study of ochratoxin toxicity in hens. Poultry Science, v. 50, p.1855-1859, 1971.

6 CONAB. Companhia Nacional de Abastecimento. Revista Indicadores da Agropecuária, Brasília, v.10, n.1, p.17, jan. 2001.

7 CORRÊA,T. B. S.; FARIA, A. X.; ANDERSEN, P. M. Qualidade sanitária do milho importado quanto à ocorrência de Aflatoxina B1, B2, G1, G2 e Ocratoxina A. In: ENCONTRO NACIONAL DE MICOTOXINAS, 1., 1998, Florianópolis. Anais... Florianópolis: UFSC/Departamento de Ciência e Tecnologia dos Alimentos, 1998. p. 116.

8 CRUZ, L.C.H. Características gerais das micotoxinas e micotoxicoses. In: SIMPÓSIO INTERNACIONAL SOBRE MICOTOXINAS E MICOTOXICOSE EM AVES. Anais... Curitiba: APINCO, 1995. p. 1-13.

9 DIRHEIMER, G. Mechanistic approaches to ochratoxin toxicity. Food Addit. Contam., v. 13, Suppl., p. 45-48, 1996.

10 ELISSALDE, M. H. et al. Effect of ochratoxin A on salmonellachalenged broiler chicks. Poultry Science, v. 73, p. 1241-1248, 1994.

11 FRAGA, M. E.; DIREITO, G. M.; SANTANA, D. M. N.; BARROS, G. C.; ROSA, C. R. Determinação pro cromatografia em camada delgada de aflatoxina (B1 e M1) e aflatoxicol em ovos destinados ao 
comércio. Revista Brasileira de Medicina Veterinária, Rio de Janeiro, v.18, n.4, p.172-175, jul./ago. 1996.

12 GIBSON, R. M. et al. Impact of L-phenylalanine supplementation on the performance of three-week-old broilers fed diets containing ochratoxin A. Effects on body weight, feed conversion, relative organ weight, and mortality. Poultry Science, v. 69, p.414-419, 1990.

13 LAZZÁRI, F. A. Umidade, fungos e micotoxinas na qualidade de sementes, grãos e rações. 2. ed. Curitiba: Ed. do Autor, 1997. $134 \mathrm{p}$.

14 LORENZINI, G. Presença de aflatoxina no alimento, cama e fígado de frango de corte e sua correlação com os parâmetros de produção. Porto Alegre, 1997. 91 p. Dissertação (Mestrado), Universidade Federal do Rio Grande do Sul.

15 MERKLEY, J. W. et al. Hepatic fatty acid profiles in aflatoxin-exposed broiler chickens. Poultry Science, v. 66, p. 59-67, 1987.

16 SABINO, M. Micotoxinas em produtos de origem animal: tecidos hepáticos e renais. In: SCUSSEL, V. M. Atualidades em micotoxinas e armazenamento de grãos. Florianópolis: Ed. da autora, 2000. p.140-149.

17 SANTÚRIO, J. M. Micotoxinas na produtividade avícola: tipos, seus efeitos e como detectá-las e prevení-las. In: CONFERÊNCIA APINCO DE CIÊNCIA E TECNOLOGIA AVÍCOLAS, 1997, São Paulo. Anais... São Paulo: FACTA, 1997. p. 224-257.

18 SALLE, C.T.P. et al. Detecção de Aflatoxina B1 no organismo de frango de corte através do emprego de ensaio-imunoenzimático utilizando anticorpos monoclonais (ELISA). In: CONGRESSO LATINOAMERICANO DE MICOTOXINAS, 1.; ENCONTRO NACIONAL DE MICOTOXINAS, 8., 1994, Rio de Janeiro. Anais... Rio de Janeiro: Centro de Micologia e Micotoxicologia, UFRRJ, 1994. p. 98-102.

19 SALLE, C. T. P. et al. Detecção de ocratoxina $A$ em víscera de frango de corte através do emprego de ensaio-imunoenzimático. $\mathbf{R}$. Bras. Med. Vet., v. 22, n. 1, p. 23-26, 2000.

20 SCOTT, P. M. Mycotoxins. J. Assoc. Anal. Chem. Intern., n.76, p.112, 1993.

21 SCUSSEL, V. M. Micotoxinas em alimentos. Florianópolis: Insular, 1998a. 110p. 
22 SCUSSEL,V. M. Vantagens e desvantagens do uso de TLC, HPLC e HPTLC. In: ENCONTRO NACIONAL DE MICOTOXINAS, 1998, Florianópolis. Anais... Florianópolis: UFSC/Departamento de Ciência e Tecnologia dos Alimentos, 1998b. p.13.

23 SFOGGIA, M.V.B.; SALLE, C.T.P. Ocratoxins detection in feedstuffs and poultry kidneys and your correlation with production parameters in a poultry company in Rio Grande do Sul. Arq. Fac. Vet. UFRGS, Porto Alegre, v.26, n.2, p.106-108, 1998.

24 SILVA, S. C., OLIVEIRA, J. N.; CALDAS, E. D. Aflatoxinas em alimentos comercializados no Distrito Federal de 1985 a 1995. R. Inst. Adolfo Lutz, São Paulo, v. 56, n.2, p.49-52, jul./dez. 1996.

25 SOARES, L.V.S.; RODRIGUEZ-AMAYA, D.B. Survery of aflatoxins, ochratoxins $A$, zearalenone and sterigmatocystin in some Brazilian food by using multi-toxin thin-layer chromatographic method. J. Assoc. Off. Anal. Chem., n.72, v.1, p.22-26, 1989.

26 VASANTHI, S.; BHAT, R. V. Mycotoxins in foods-occurrence, health, economic significance, food control measures. Ind. J. Med. Res., v. 108, p. 212-224, 1998.

27 VIEIRA, S. L. Micotoxinas e produção de ovos. In: SIMPÓSIO INTERNACIONAL SOBRE MICOTOXINAS E MICOTOXICOSE EM AVES. Anais... Curitiba: APINCO, 1995. p. 65-80.

28 VILAR, E.A.; STAMFORD, T.L.M. Micotoxinas: uma ameaça à saúde. Ciência Veterinária nos Trópicos, Recife, v.1, n.2, p. 130134, set./dez. 1998.

\section{Agradecimentos}

À Fundação de Amparo à Ciência e Tecnologia do Estado de Pernambuco (FACEPE) e ao Conselho Nacional de Pesquisas (CNPq) pelo apoio financeiro. 